\title{
Screen-time, obesity, ageing and disability: findings from 91266 participants in the 45 and Up Study
}

\author{
Emily Banks ${ }^{1,2, *}$, Louisa Jorm ${ }^{2,3}$, Kris Rogers ${ }^{2}$, Mark Clements ${ }^{1}$ and Adrian Bauman ${ }^{4}$ \\ ${ }^{1}$ National Centre for Epidemiology and Population Health, Australian National University, Acton, ACT 0200, \\ Australia: ${ }^{2}$ The Sax Institute, Broadway, NSW 2007, Australia: ${ }^{3}$ School of Medicine, University of Western \\ Sydney, Campbelltown Campus, Penrith South DC, NSW 1797, Australia: ${ }^{4}$ Prevention Research Collaboration, \\ School of Public Health, University of Sydney, NSW 2006, Australia
}

Submitted 13 October 2009: Accepted 23 February 2010: First published online 22 April 2010

\begin{abstract}
Objective: To assess the relationship between obesity and sedentary behaviours, such as watching television or using a computer ('screen-time'), and describe how this relationship varies between population subgroups.

Design: Cross-sectional analysis of the relationship between obesity (BMI $\left.\geq 30 \mathrm{~kg} / \mathrm{m}^{2}\right)$ and screen-time, adjusted for age, sex, income and education and compared according to a range of personal characteristics.

Setting: New South Wales, Australia.

Subjects: A total of 91266 men and women aged 45 years and above from the general population of New South Wales in 2006-2007 and providing self-reported information on height and weight and other factors.

Results: Obesity prevalence was $21.4 \%$. Compared to individuals with $<2 \mathrm{~h}$ of daily screen-time, the adjusted relative risks (RR) of obesity were 1.35 (95\% CI $1 \cdot 26,1 \cdot 44), 1 \cdot 70(95 \%$ CI $1.59,1 \cdot 82), 1 \cdot 94(95 \%$ CI $1 \cdot 81,2 \cdot 08)$ and $1.92(95 \%$ CI $1 \cdot 80,2 \cdot 06$ ) for $2-3,4-5,6-7$ and $\geq 8 \mathrm{~h}$, respectively. The increase in obesity with increasing screen-time was similar within categories of overall physical activity, but was attenuated in those in full-time paid work, compared to non-workers ( $P$ for interaction $<0 \cdot 0001$ ). Among non-workers, the overall obesity RR per $2 \mathrm{~h}$ increase in daily screen-time was $1 \cdot 23(95 \%$ CI $1 \cdot 21,1 \cdot 25)$ and was significantly elevated in all groups examined, ranging from $1 \cdot 16$ to $1 \cdot 31$ according to sex, level of age, education, income, smoking and fruit consumption. The RR did not differ significantly according to overall physical activity, region of residence and alcohol and vegetable consumption, but was substantially lower in disabled $v$. not-disabled individuals ( $P$ for interaction $<0 \cdot 0001$ ).

Conclusions: Obesity increases with increasing screen-time, independent of purposeful physical activity. This was observed in all population groups examined, although it is attenuated in full-time workers and disabled individuals.
\end{abstract}

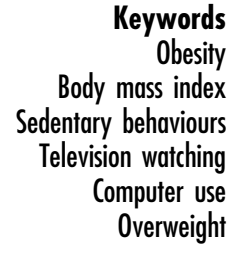

Increasing obesity rates present major challenges to health, locally and globally. Inadequate energy expenditure is a component of obesogenesis, as part of an overall energy imbalance, but evidence regarding the positive and negative contributions of specific types of activities is limited. Recent evidence indicates that sedentary behaviours, such as watching television, using a computer and sitting, are at least as important as purposeful moderate or vigorous leisure-time physical activity in influencing $\mathrm{BMI}^{(1-10)}$. However, it is not known whether this relationship holds true across the whole population, or whether there are specific subgroups that are more or less vulnerable to the adverse effects of sedentary behaviour. In particular, since physical activity levels tend to decline with age ${ }^{(9)}$ and physical disability, it is important to understand the influence of sedentary behaviours in the elderly $^{(2)}$ and functionally impaired. Gradients in the relationship between sedentary behaviours and obesity according to socio-economic and lifestyle factors could potentially exacerbate existing inequalities in health. Finally, work-related sitting time has been found to have a strong influence on patterns of inactivity, and the need to consider both work-related sedentary behaviour and physical activity in promoting a healthy BMI has been highlighted $^{(11)}$.

Current mass-reach campaigns to reduce obesity focus almost exclusively on increasing periodic purposeful leisure-time physical activity, rather than decreasing time spent in sedentary activities. Reliable information on the relationship between sedentary activities and obesity, 
together with evidence regarding the need to target specific groups, will be important in planning for future effective interventions to reduce obesity.

The present study examines the relationship between BMI and time spent watching television or using a computer ('screen-time') and other markers of physical activity and inactivity in a large cohort of Australian adults aged 45 years and above that includes sufficient numbers of participants to allow quantification of this relationship in different population subgroups. Particular attention is given to how observed relationships vary with age, disability, work status, socio-economic and lifestyle factors.

\section{Methods}

\section{Study population}

The 45 and Up Study is a large-scale study of healthy ageing of men and women aged 45 years and above from the general population of New South Wales, Australia. It is described in detail elsewhere ${ }^{(12)}$. Briefly, individuals aged 45 years and above were sampled from the Medicare Australia database, which provides virtually complete coverage of the general population, and joined the study by completing a postal questionnaire and providing written consent for long-term follow-up of their health. There was twofold oversampling of individuals aged 80 years and above and those resident in rural areas.

Recruitment into the study began in February 2006 and these analyses relate to 91266 participants joining the study up to the end of April 2008.

\section{Data}

All variables were derived from self-reported data from the 45 and Up Study questionnaire (available at www. 45andUp.org.au), apart from the measure of remoteness of residence, which was assigned according to the mean Accessibility Remoteness Index of Australia Plus ${ }^{(13)}$ score for the postcode of the participant's residential address, as recorded by Medicare Australia and categorised as resident in a major city or regional or remote area.

Participants' overall level of physical activity was classified according to their responses to elements of the Active Australia Questionnaire ${ }^{(14,15)}$, comprising information on number of weekly sessions (of any duration) of moderate and vigorous physical activity and episodes of walking for longer than $10 \mathrm{~min}$. A weighted weekly average number of sessions was calculated for each participant by adding the total number of sessions, with vigorous activity sessions receiving twice the weighting of moderate activity or walking sessions ${ }^{(16)}$, and was categorised as zero to three, four to nine, ten to seventeen and eighteen or more sessions per week. The use of weekly sessions of activity rather than a sum of the total time spent in physical activity allowed reconciliation across different versions of the study questionnaire; slight changes to questionnaire layout and wording across versions affected the reporting of time spent in physical activity, but not sessions of physical activity. The predictive value of total (MET-adjusted) weekly sessions for meeting the current physical activity recommendations (defined as a total of $150 \mathrm{~min}$ of physical activity in five or more sessions per week ${ }^{(14,15)}$ ) was tested with a receiver operating characteristic curve. The area under the curve was $85.6 \%$, which indicates that weekly sessions have good predictive value for sufficient physical activity.

Total daily screen-time, sitting time and standing time were classified according to the participant's response to the question 'About how many hours in each $24 \mathrm{~h}$ day do you usually spend doing the following: watching television or using a computer; sitting; sleeping; standing.' These variables were categorised as $0-1,2-3,4-5,6-7$ and $\geq 8 \mathrm{~h} / \mathrm{d}$, except for sleeping, which was categorised as $0-5,6-7,8,9-10$ and $\geq 11 \mathrm{~h} / \mathrm{d}$.

Self-reported weight and height were used to calculate participants' BMI, as their weight in kilograms divided by the square of their height in metres $\left(\mathrm{kg} / \mathrm{m}^{2}\right)$. BMI was categorised according to the WHO criteria as underweight $\left(B M I<18.5 \mathrm{~kg} / \mathrm{m}^{2}\right)$, healthy weight $(\mathrm{BMI}=18.5-24.9 \mathrm{~kg} /$ $\mathrm{m}^{2}$ ), overweight (BMI $\left.=25 \cdot 0-29 \cdot 9 \mathrm{~kg} / \mathrm{m}^{2}\right)$ and obese $\left(\mathrm{BMI} \geq 30 \mathrm{~kg} / \mathrm{m}^{2}\right)^{(17)}$.

Individuals reporting needing assistance with daily tasks because of long-term illness or disability were considered to have a major disability. Functional capacity was measured using the Medical Outcomes Score Physical Functioning (equivalent to the physical functioning subscore from the SF-36) with those with a score of 100 considered to have no functional limitations and those with scores of 90-99, 60-89 and 0-59 having minor, moderate and severe limitations, respectively.

Individuals were excluded from the analyses if they were missing data on height ( $n$ 5618), weight ( $n$ 4172) or physical activity ( $n$ 4869), leaving 91266 participants.

\section{Statistical metbods}

The relationship between overall physical activity, screentime, sitting time and standing time and a range of personal characteristics was examined, as well as the correlation between the individual measures of physical activity and inactivity.

The proportion of the study population classified as obese according to screen-time, sitting time and standing time was examined overall and separately according to a range of factors. Relative risks (RR; prevalence ratios) and 95\% CI for obesity according to screen-time, sitting time and standing time were estimated by generalised linear models, with a log link and binomial distribution ${ }^{(18)}$, adjusting where appropriate for age (in 5-year age groups), sex (male and female), income (pre-tax total annual household income $<\$ 20000, \$ 20000-\$ 49999$ and $\geq \$ 50000$ Australian dollars) and educational attainment (less than secondary school graduation, secondary school 
graduation, post-secondary school certificate or diploma and tertiary graduate). Sensitivity analyses were conducted examining the effect on the screen-time model of additional adjustment for smoking (current, past and never) alcohol consumption (zero to four, five to eleven, twelve to twenty and twenty-one or more alcoholic drinks per week), fruit (less than two and two or more servings per day) and vegetable consumption (less than five and five or more servings per day), functional capacity (as categorised above) and disability (assistance required for daily tasks, no assistance required for daily tasks). Where appropriate, categories were included for missing values. Tests for linear trend were performed by treating selected ordinal variables as continuous. To test for interaction using multiple screentime categories, we used a likelihood ratio test comparing the model with and without the interaction term. The weighted least-squares method was used to compare the RR of obesity per $2 \mathrm{~h}$ of additional daily screen-time in different study subgroups.

We examined how much of any effect of a specific sedentary behaviour on obesity was attributable to differences in total physical activity level, first by adding it to the regression model and then by reporting the relationship between screen-time and obesity in different categories of overall physical activity. We also examined how much of the effect of certain sedentary behaviours could be attributed to the effect of other sedentary behaviours, using mutual adjustment.

People in paid work have different patterns of screentime compared to those who are not working, due to the amount of work that requires use of computers; similar issues apply to patterns of sitting and standing time. We hypothesised, a priori, that the effects of screen-time and other sedentary behaviours on obesity would vary according to paid work status, and examined this statistically. Overall, $29 \cdot 5 \%$ of the cohort was in full-time paid work and work status related closely to income and education. The proportion of people in paid work varied markedly by age; only $0.6 \%$ of men and $0.3 \%$ of women aged 70 years and above reported being in paid work. Initial analyses examined the relationship between obesity and physical activity, screen-time, sitting time and sleeping time in the whole cohort. Because of a lack of elderly people in paid work, subsequent analysis were restricted to those not in paid work, in order to examine the relationship between screen-time and obesity according to a participant's age, sex, income, education, region of residence, smoking status and level of disability.

The population attributable fraction was calculated using the relevant adjusted RR and the exposure prevalence among those with obesity $\left(\mathrm{p}_{c}\right)$ in the formula $\mathrm{AF}_{p}=\mathrm{p}_{c}(\mathrm{RR}-1) / \mathrm{RR}^{(19)}$. CI was calculated using a complementary-log transformation ${ }^{(19)}$.

All analyses were carried out in SAS statistical software package version $9 \cdot 13$ (SAS Institute, Cary, NC, USA). All statistical tests were two-sided, using a significance level of $P<0 \cdot 05$. Because of the large sample size, conclusions were based on both significance and the effect size.

\section{Results}

Of 91266 participants, $34235(37 \cdot 5 \%, 95 \%$ CI 37•4, 37·6) were classified as being of healthy weight, $1334(1 \cdot 4 \%$, $95 \%$ CI $1 \cdot 1,1 \cdot 8)$ were underweight, $36156(39 \cdot 6 \%, 95 \%$ CI $39 \cdot 5,39 \cdot 7)$ were overweight but not obese and 19541 $(21 \cdot 4 \%, 95 \%$ CI $21 \cdot 4,21 \cdot 5)$ were obese. The average total sessions of physical activity per week was $13 \cdot 0$ (SD 19.60), with $4 \cdot 0 \%(95 \%$ CI $3 \cdot 9,4 \cdot 1 \%)$ reporting no sessions of physical activity and $19 \cdot 3 \%$ (95\% CI $19 \cdot 1,19 \cdot 6)$ reporting eighteen or more sessions per week. Overall, $7 \cdot 4 \%$ of participants reported a screen-time of $<2 \mathrm{~h} / \mathrm{d}, 40.6 \%$ reported $2 \cdot 0-3 \cdot 9 \mathrm{~h}, 30 \cdot 6 \%$ reported $4 \cdot 0-5 \cdot 9 \mathrm{~h}, 10 \cdot 4 \%$ reported $6-7 \cdot 9 \mathrm{~h}$ and $11.0 \%$ reported $\geq 8 \mathrm{~h} / \mathrm{d}$, with an overall average of $4 \cdot 1(\mathrm{SD} 2 \cdot 51) \mathrm{h} / \mathrm{d}$.

Individuals who had more screen-time were more likely to be male, current smokers and to eat fewer servings of fruit and vegetables per week than individuals with less screen-time ( $P$ for trend $<0 \cdot 001$; Table 1 ). Screen-time was particularly high in those who were of working age, with higher income and higher education levels ( $P$ for trend $<0 \cdot 001$; Table 1 ). Those reporting a significant disability and those with lower functional capacity had longer overall screen-time ( $P$ for trend $<0 \cdot 001$; Table 1 ). Number of hours of daily screen-time was poorly correlated with number of weekly sessions of physical activity $(r=-0 \cdot 06,95 \%$ CI $-0 \cdot 07,-0 \cdot 05)$, but was moderately well correlated with sitting hours per day $(r=0 \cdot 44,95 \%$ CI $0 \cdot 44,0 \cdot 47)$ and inversely related to hours spent standing per day $(r=-0 \cdot 25,95 \%$ CI $-0 \cdot 28$, $-0 \cdot 23)$.

\section{Screen-time, otber sedentary bebaviours and obesity}

Table 2 shows a steady increase in the risk of obesity with increasing screen-time, with $15 \cdot 0 \%$ of those with a screen-time of $0-1 \cdot 9 \mathrm{~h} / \mathrm{d}$ being obese compared to $27 \cdot 6 \%$ of those with 8 or more screen-hours per day. The age-, sex-, income- and education-adjusted RR of obesity was $1.92(95 \%$ CI $1.80,2.06)$ in those with 8 or more compared to those with 0-1 daily screen-hours, and there was a significant trend of increasing obesity with increasing screen-time ( $P$ for trend $<0 \cdot 001$ ). When screen-time was treated as a continuous variable, the correlation between screen-time and BMI was 0.12 (95\% CI 0.12, 0.13; $P<0 \cdot 001)$. A similar but less dramatic relationship was observed between overall sitting time and obesity, and there was an inverse relationship between time spent standing and obesity (Table 2).

The prevalence of obesity decreased with increasing sessions of physical activity per week, such that those with eighteen and more sessions per week had half the 
Table 1 Characteristics of the study population according to total daily screen-time

\begin{tabular}{|c|c|c|c|c|c|c|c|c|c|c|c|}
\hline & \multicolumn{10}{|c|}{ Hours of screen-time per day } & \multirow[b]{3}{*}{$P$ for trend } \\
\hline & \multicolumn{2}{|c|}{$0-1 \cdot 9 \mathrm{~h}$} & \multicolumn{2}{|c|}{$2 \cdot 0-3 \cdot 9 \mathrm{~h}$} & \multicolumn{2}{|c|}{$4 \cdot 0-5 \cdot 9 \mathrm{~h}$} & \multicolumn{2}{|c|}{$6 \cdot 0-7 \cdot 9 \mathrm{~h}$} & \multicolumn{2}{|c|}{$>8 \mathrm{~h}$} & \\
\hline & $\%$ & $n$ & $\%$ & $n$ & $\%$ & $n$ & $\%$ & $n$ & $\%$ & $n$ & \\
\hline Male & $46 \cdot 8$ & 3534 & $47 \cdot 4$ & 19071 & $47 \cdot 9$ & 14535 & $49 \cdot 4$ & 5007 & $48 \cdot 5$ & 5178 & $<0.001$ \\
\hline Urban resident & $42 \cdot 4$ & 3195 & $41 \cdot 2$ & 16633 & $42 \cdot 2$ & 12803 & $45 \cdot 5$ & 4601 & $52 \cdot 1$ & 5557 & $<0.001$ \\
\hline Tertiary educated & $27 \cdot 1$ & 2008 & $23 \cdot 1$ & 9099 & $19 \cdot 5$ & 5797 & $24 \cdot 2$ & 2415 & $31 \cdot 7$ & 3331 & $<0.001$ \\
\hline Annual income $\geq \$ A U 70000$ & $23 \cdot 5$ & 1651 & $22 \cdot 1$ & 8455 & $17 \cdot 8$ & 5099 & $25 \cdot 7$ & 2478 & $42 \cdot 4$ & 4334 & $<0.001$ \\
\hline In full-time paid work & $32 \cdot 1$ & 2343 & $27 \cdot 4$ & 10752 & $19 \cdot 5$ & 5778 & $28 \cdot 8$ & 2860 & $57 \cdot 2$ & 6009 & $<0.001$ \\
\hline Current smoker & $7 \cdot 5$ & 564 & $6 \cdot 4$ & 2586 & $7 \cdot 3$ & 2230 & $8 \cdot 4$ & 853 & $9 \cdot 0$ & 958 & $<0.001$ \\
\hline Disabled & $5 \cdot 6$ & 421 & $4 \cdot 1$ & 1630 & $5 \cdot 5$ & 1654 & $7 \cdot 0$ & 709 & $6 \cdot 5$ & 688 & $<0.001$ \\
\hline \multirow[t]{2}{*}{ Functional capacity (in lower third) } & $27 \cdot 5$ & 1823 & $28 \cdot 8$ & 10390 & $36 \cdot 1$ & 9891 & $37 \cdot 2$ & 3437 & $29 \cdot 9$ & 2941 & $<0.001$ \\
\hline & Mean & SD & Mean & SD & Mean & SD & Mean & SD & Mean & SD & \\
\hline Age (years) & $62 \cdot 3$ & $11 \cdot 1$ & $63 \cdot 2$ & $12 \cdot 1$ & $64 \cdot 7$ & $11 \cdot 3$ & $62 \cdot 5$ & $10 \cdot 9$ & $58 \cdot 1$ & $10 \cdot 7$ & $<0.001$ \\
\hline $\mathrm{BMl}\left(\mathrm{kg} / \mathrm{m}^{2}\right)$ & $25 \cdot 5$ & $4 \cdot 8$ & $26 \cdot 4$ & $4 \cdot 5$ & $27 \cdot 1$ & $4 \cdot 5$ & $27 \cdot 7$ & $4 \cdot 8$ & $27 \cdot 7$ & $5 \cdot 2$ & $<0.001$ \\
\hline Alcohol consumption (g/week) & $7 \cdot 0$ & $9 \cdot 9$ & $7 \cdot 3$ & $11 \cdot 1$ & $7 \cdot 1$ & $9 \cdot 8$ & $7 \cdot 0$ & $9 \cdot 8$ & $7 \cdot 3$ & $9 \cdot 9$ & 0.986 \\
\hline Vegetable intake (servings/d) & $3 \cdot 6$ & $2 \cdot 7$ & $3 \cdot 8$ & $2 \cdot 7$ & 3.9 & $2 \cdot 7$ & $3 \cdot 7$ & $2 \cdot 7$ & $3 \cdot 5$ & $2 \cdot 6$ & $<0.001$ \\
\hline Fruit intake (servings/d) & $2 \cdot 4$ & $1 \cdot 6$ & $2 \cdot 4$ & $1 \cdot 7$ & $2 \cdot 3$ & $1 \cdot 5$ & $2 \cdot 3$ & $1 \cdot 5$ & $2 \cdot 2$ & $1 \cdot 5$ & $<0.001$ \\
\hline Physical activity (sessions/week) & $14 \cdot 9$ & $19 \cdot 4$ & $13 \cdot 8$ & $22 \cdot 5$ & $12 \cdot 6$ & $20 \cdot 6$ & $11 \cdot 5$ & $18 \cdot 9$ & $10 \cdot 9$ & $15 \cdot 2$ & $<0.001$ \\
\hline Sitting $(\mathrm{h} / \mathrm{d})$ & $4 \cdot 2$ & $3 \cdot 1$ & $4 \cdot 6$ & $3 \cdot 1$ & $5 \cdot 5$ & $2 \cdot 7$ & $6 \cdot 7$ & $2 \cdot 5$ & $8 \cdot 8$ & $2 \cdot 7$ & $<0.001$ \\
\hline Standing $(h / d)$ & $5 \cdot 7$ & $3 \cdot 3$ & $5 \cdot 3$ & $4 \cdot 1$ & $4 \cdot 6$ & $3 \cdot 5$ & $3 \cdot 8$ & $3 \cdot 0$ & $2 \cdot 9$ & $2 \cdot 6$ & $<0.001$ \\
\hline Sleeping $(h / d)$ & $7 \cdot 6$ & $1 \cdot 4$ & $7 \cdot 7$ & 1.9 & $7 \cdot 7$ & $1 \cdot 4$ & $7 \cdot 6$ & $1 \cdot 4$ & $7 \cdot 5$ & $1 \cdot 4$ & $<0.001$ \\
\hline
\end{tabular}

risk of obesity of people reporting zero to three sessions per week (Table 2). Following adjustment for sessions of physical activity, the RR of obesity with increasing screen-time was attenuated slightly to 1.69 for $\geq 8 \mathrm{~h} / \mathrm{d}$ screen-time compared to that of $0-1 \mathrm{~h} / \mathrm{d}$ (Table 2 ). The OR did not change materially (i.e. $<5 \%$ ) with additional adjustment for smoking, alcohol consumption, fruit and vegetable consumption and disability. A significant positive association between obesity and sitting time and a significant negative association with standing time persisted following adjustment.

Although levels of obesity were lower in individuals with higher levels of overall physical activity, increasing screen-time was accompanied by increasing obesity within each category of physical activity, and the shape of the obesity/screen-time dose-response curve was similar for each level of reported physical activity (Fig. 1). There was minor heterogeneity in the effect of screen-time on obesity between different categories, with a slight attenuation in the association between screen-time and obesity among those with the lowest overall levels of physical activity $\left(\chi_{12}^{2}\right.$ for interaction $\left.=28 \cdot 3, P=0 \cdot 005\right)$. Considering screen-time and overall physical activity together, individuals who were most active but had the highest screen-times had risks of obesity similar to those of individuals who were the least active but had the lowest screen-times; their RR of obesity were $1.72(95 \%$ CI $1 \cdot 46,2 \cdot 03)$ and $2 \cdot 05$ (95\% CI $1 \cdot 71,2 \cdot 45)$, respectively, compared to individuals with the lowest screen-times and the highest physical activity levels. Those with the highest screen-time and lowest overall physical activity level had an RR of obesity of $3 \cdot 04$ (95\% CI $2 \cdot 63,2 \cdot 45)$, compared to individuals with the lowest screen-time and highest physical activity levels (Fig. 1).
The effect of screen-time on obesity was attenuated following adjustment for sitting time, compared to individuals with $0-1 \mathrm{~h}$ screen-time/d; the RR of obesity were 1.01 (95\% CI $0 \cdot 93,1 \cdot 1), 1 \cdot 04(95 \%$ CI $0 \cdot 96,1 \cdot 12), 1 \cdot 05$ (95\% CI $0 \cdot 97$, $1 \cdot 14)$ and $1 \cdot 13(95 \%$ CI $1 \cdot 04,1 \cdot 23)$ for those with a screentime of $2-3,4-5,6-7$ and $\geq 8 \mathrm{~h} / \mathrm{d}$, respectively, adjusting for age, sex, income, education and sitting time.

\section{The relationship between screen-time and obesity according to work status}

People in paid work reported an average screen-time of $4.7(\mathrm{sD} 2 \cdot 1) \mathrm{h} / \mathrm{d}$, while those in part-time paid work and those not in paid work reported $3 \cdot 8$ (SD 2.3) and $3 \cdot 9$ (SD $2 \cdot 1$ ) h/d, respectively. The adjusted RR of obesity increased significantly with increasing screen-time regardless of work status (Fig. 2); however, the effect of screen-time was significantly and substantially greater among those who were not in paid work compared to those in paid work ( $\chi_{4}^{2}$ for interaction $=194 \cdot 4, \quad P<$ $0 \cdot 00001$ for comparison of the effects in Fig. 2). The RR of obesity per $2 \mathrm{~h}$ of additional screen-time was 1.08 (95\% CI $1 \cdot 06,1 \cdot 10)$ for those in full-time work, $1 \cdot 15$ (95\% CI $1 \cdot 12,1 \cdot 19)$ for those in part-time work and $1 \cdot 23$ (95\% CI $1 \cdot 21,1 \cdot 25)$ for those not in paid work $\left(\chi_{2}^{2}\right.$ for interaction $=161 \cdot 9, P<0 \cdot 00001)$.

\section{The relationship between screen-time and obesity according to age, disability and otber factors}

The analyses were restricted to those who were not in paid work to permit valid comparisons of the effect of screen-time across the full age range. The age-, sex-, income- and education-adjusted RR of obesity per $2 \mathrm{~h}$ increase in daily screen-time are shown separately according to a variety of factors in Fig. 3. On average, 
Table 2 Relative risk of obesity according to sedentary behaviours and physical activity

\begin{tabular}{|c|c|c|c|c|c|c|c|c|}
\hline \multirow[b]{2}{*}{ Total } & \multirow{2}{*}{$\begin{array}{c}\text { Total } \\
n\end{array}$} & \multirow{2}{*}{$\begin{array}{c}\text { Obese } \\
\%\end{array}$} & \multicolumn{2}{|c|}{ Age- and sex-adjusted } & \multicolumn{2}{|c|}{ Adjusted } & \multicolumn{2}{|c|}{ Adjusted } \\
\hline & & & $\mathrm{RR}$ & $95 \% \mathrm{Cl}$ & $\mathrm{RR}$ & $95 \% \mathrm{Cl}^{*}$ & $\mathrm{RR}$ & $95 \% \mathrm{Clt}$ \\
\hline \multicolumn{9}{|l|}{ Screen-time $(\mathrm{h} / \mathrm{d})$} \\
\hline $0-1$ & 6943 & $15 \cdot 0$ & $1 \cdot 00$ & & $1 \cdot 00$ & & $1 \cdot 00$ & \\
\hline $2-3$ & 37376 & $18 \cdot 1$ & $1 \cdot 36$ & $1 \cdot 28,1 \cdot 46$ & $1 \cdot 35$ & $1 \cdot 26,1 \cdot 44$ & $1 \cdot 23$ & $1 \cdot 15,1 \cdot 32$ \\
\hline $4-5$ & 28105 & $23 \cdot 1$ & $1 \cdot 76$ & $1 \cdot 65,1 \cdot 88$ & $1 \cdot 70$ & $1 \cdot 59,1 \cdot 82$ & 1.51 & $1 \cdot 41,1 \cdot 62$ \\
\hline $6-7$ & 9443 & $27 \cdot 2$ & 1.97 & $1 \cdot 84,2 \cdot 12$ & 1.94 & $1 \cdot 81,2 \cdot 08$ & $1 \cdot 71$ & $1 \cdot 59,1 \cdot 85$ \\
\hline$\geq 8$ & 9927 & $27 \cdot 6$ & 1.91 & $1 \cdot 78,2 \cdot 05$ & 1.92 & $1 \cdot 80,2 \cdot 06$ & $1 \cdot 69$ & $1 \cdot 57,1 \cdot 82$ \\
\hline$P$ for trend & & & $<0.001$ & & $<0.001$ & & $<0.001$ & \\
\hline \multicolumn{9}{|c|}{ Time spent sitting $(\mathrm{h} / \mathrm{d})$} \\
\hline $0-1$ & 3932 & $18 \cdot 3$ & $1 \cdot 00$ & & $1 \cdot 00$ & & $1 \cdot 00$ & \\
\hline $2-3$ & 19716 & $18 \cdot 9$ & $1 \cdot 05$ & $0.97,1.13$ & $1 \cdot 07$ & $0.99,1.15$ & 1.04 & $0.95,1.12$ \\
\hline $4-5$ & 25741 & $20 \cdot 6$ & $1 \cdot 15$ & $1 \cdot 07,1 \cdot 24$ & $1 \cdot 17$ & $1 \cdot 08,1 \cdot 26$ & $1 \cdot 13$ & $1 \cdot 05,1 \cdot 23$ \\
\hline $6-7$ & 17445 & $22 \cdot 1$ & $1 \cdot 23$ & $1 \cdot 14,1 \cdot 33$ & $1 \cdot 28$ & $1 \cdot 19,1 \cdot 38$ & $1 \cdot 22$ & $1 \cdot 12,1 \cdot 32$ \\
\hline$\geq 8$ & 21714 & $24 \cdot 6$ & $1 \cdot 35$ & $1 \cdot 25,1 \cdot 45$ & $1 \cdot 44$ & $1 \cdot 33,1.54$ & $1 \cdot 33$ & $1 \cdot 23,1 \cdot 44$ \\
\hline$P$ for trend & & & $<0.001$ & & $<0.001$ & & $<0.001$ & \\
\hline \multicolumn{9}{|c|}{ Time spent standing $(\mathrm{h} / \mathrm{d})$} \\
\hline $0-1$ & 13553 & $22 \cdot 6$ & $1 \cdot 00$ & $1 \cdot 00$ & $1 \cdot 00$ & & & \\
\hline $2-3$ & 22774 & $23 \cdot 1$ & 1.03 & $0.99,1.07$ & $1 \cdot 02$ & $0.99,1.06$ & $1 \cdot 02$ & $0.98,1.06$ \\
\hline $4-5$ & 16781 & $21 \cdot 9$ & 0.99 & $0.95,1.04$ & 0.97 & $0.93,1.01$ & 0.97 & $0.93,1.01$ \\
\hline $6-7$ & 12025 & $20 \cdot 4$ & 0.92 & $0.88,0.96$ & $0 \cdot 89$ & $0.85,0.93$ & $0 \cdot 91$ & $0.87,0.96$ \\
\hline$\geq 8$ & 18961 & $18 \cdot 7$ & $0 \cdot 81$ & $0 \cdot 77,0.84$ & $0 \cdot 76$ & $0 \cdot 73,0.80$ & $0 \cdot 80$ & $0.76,0.84$ \\
\hline$P$ for trend & & & $<0.001$ & & $<0.001$ & & $<0.001$ & \\
\hline \multicolumn{9}{|c|}{ Time spent sleeping $(\mathrm{h} / \mathrm{d})$} \\
\hline $0-5 \mathrm{~h}$ & 3690 & $27 \cdot 0$ & $1 \cdot 00$ & & $1 \cdot 00$ & & 1.00 & \\
\hline $6-7 \mathrm{~h}$ & 11821 & $23 \cdot 9$ & $0 \cdot 90$ & $0.84,0.96$ & 0.94 & $0.88,1.00$ & 0.91 & $0.84,0.97$ \\
\hline $8 \mathrm{~h}$ & 59774 & $20 \cdot 1$ & $0 \cdot 74$ & $0.70,0.79$ & $0 \cdot 80$ & $0.76,0.85$ & $0 \cdot 77$ & $0.72,0.82$ \\
\hline $9-10 \mathrm{~h}$ & 15820 & $22 \cdot 3$ & $0 \cdot 85$ & $0.80,0.91$ & $0 \cdot 89$ & $0.84,0.95$ & 0.85 & $0.79,0.92$ \\
\hline$\geq 11 \mathrm{~h}$ & 1694 & $27 \cdot 7$ & 1.05 & $0.95,1 \cdot 16$ & 1.04 & $0.95,1.14$ & $1 \cdot 00$ & $0 \cdot 89,1 \cdot 12$ \\
\hline$P$ for trend & & & $<0.001$ & & $<0.001$ & & $<0.001$ & \\
\hline \multicolumn{9}{|c|}{ Sessions of physical activity per week } \\
\hline $0-3$ sessions & 12043 & $31 \cdot 5$ & $1 \cdot 00$ & & $1 \cdot 00$ & & & \\
\hline 4-6 sessions & 14750 & $25 \cdot 5$ & $0 \cdot 76$ & $0 \cdot 73,0.80$ & $0 \cdot 79$ & $0 \cdot 76,0 \cdot 82$ & & \\
\hline 7-11 sessions & 23241 & $21 \cdot 1$ & 0.64 & $0.62,0.67$ & 0.67 & $0.64,0.70$ & & \\
\hline $11-17$ sessions & 23550 & $17 \cdot 8$ & 0.53 & $0.51,0.55$ & 0.56 & $0.54,0.58$ & & \\
\hline$\geq 18$ sessions & 17607 & $16 \cdot 6$ & 0.48 & $0.46,0.51$ & 0.51 & $0.48,0.53$ & & \\
\hline$P$ for trend & & & $<0.001$ & & $<0.001$ & & & \\
\hline
\end{tabular}

*Adjusted for age, sex, income and education.

tAdjusted for age, sex, income, education and overall physical activity.

there was a $23 \%(95 \%$ CI 21, 25) increase in the risk of obesity with every $2 \mathrm{~h}$ of additional daily screen-time overall, and a significant elevation in the risk of obesity with increasing screen-time was seen in all of the population and risk factor subgroups examined (Fig. 3).

The effect of screen-time on obesity was slightly attenuated in the 45-64-year-old age group ( $\chi_{2}^{2}$ for heterogeneity $=9 \cdot 5, P=0 \cdot 009)$ compared to other ages, although this difference was not large. The effect of screen-time on obesity was slightly greater in women than in men. This RR was 1.16 (95\% CI 1.09, 1.24) among current smokers, which was significantly, although not markedly, lower than that seen among ex-smokers $(1 \cdot 22,95 \%$ CI $1 \cdot 20,1 \cdot 25)$ and never-smokers $\left(1 \cdot 25,95 \%\right.$ CI $1 \cdot 23,-1 \cdot 27 ; \chi_{2}^{2}$ for heterogeneity $=7 \cdot 2, P=0 \cdot 03)$. The association between screentime and obesity was attenuated somewhat in those with lower income, educational attainment and fruit consumption (Fig. 3); this heterogeneity remained when smokers were excluded from the analysis but was substantially reduced when individuals with severe functional limitation were excluded (data not shown). The RR of obesity with increasing screen-time did not differ significantly according to region of residence, alcohol consumption and vegetable consumption.

Although a broadly similar pattern of increasing risk of obesity with increasing screen-time was seen in those with and without a major disability, this relationship differed substantially between these groups. The RR of obesity with every $2 \mathrm{~h}$ of additional daily screen-time was $1 \cdot 23$ (95\% CI $1 \cdot 20,1 \cdot 25)$ in those not requiring help with day-to-day tasks, but was attenuated to $1 \cdot 10$ (95\% CI $1 \cdot 06,1 \cdot 14)$ in those requiring help $\left(\chi_{1}^{2}\right.$ for heterogeneity $=27 \cdot 5, P<0 \cdot 00001)$. The relative effect of screentime on obesity diminished gradually with decreasing functional capacity (Fig. 3). Those reporting requiring help for daily tasks or the lowest functional capacity category had a mean number of physical activity sessions of $8 \cdot 4$, which was significantly lower than that seen in the rest of the cohort (mean $=13 \cdot 2$ ). However, even among those reporting serious disability, people reporting a screen-time of $\geq 8 \mathrm{~h} / \mathrm{d}$ were $66 \%$ (95\% CI 34, 99), and more likely to be obese compared to those reporting $<2 \mathrm{~h}$ of daily screen-time, and the absolute level of obesity was higher in disabled participants. 


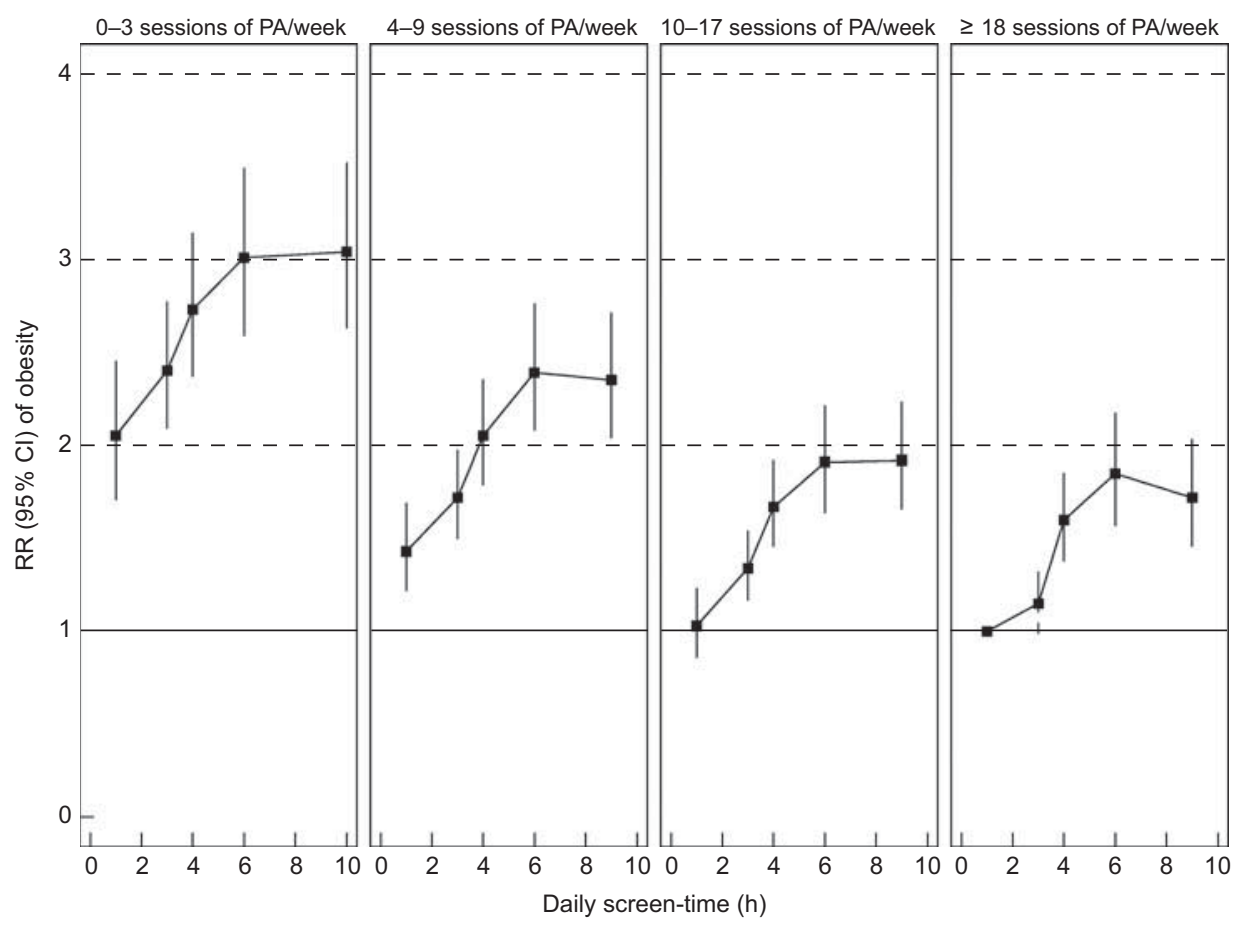

Fig. 1 Relative risk (RR) of obesity according to hours of daily screen-time, in categories of overall physical activity (PA), adjusted for age, sex, income and education (reference group: $\geq 18$ sessions of PA per week, $<2 \mathrm{~h}$ of daily screen-time); RR are plotted against the median daily screen-time for each category

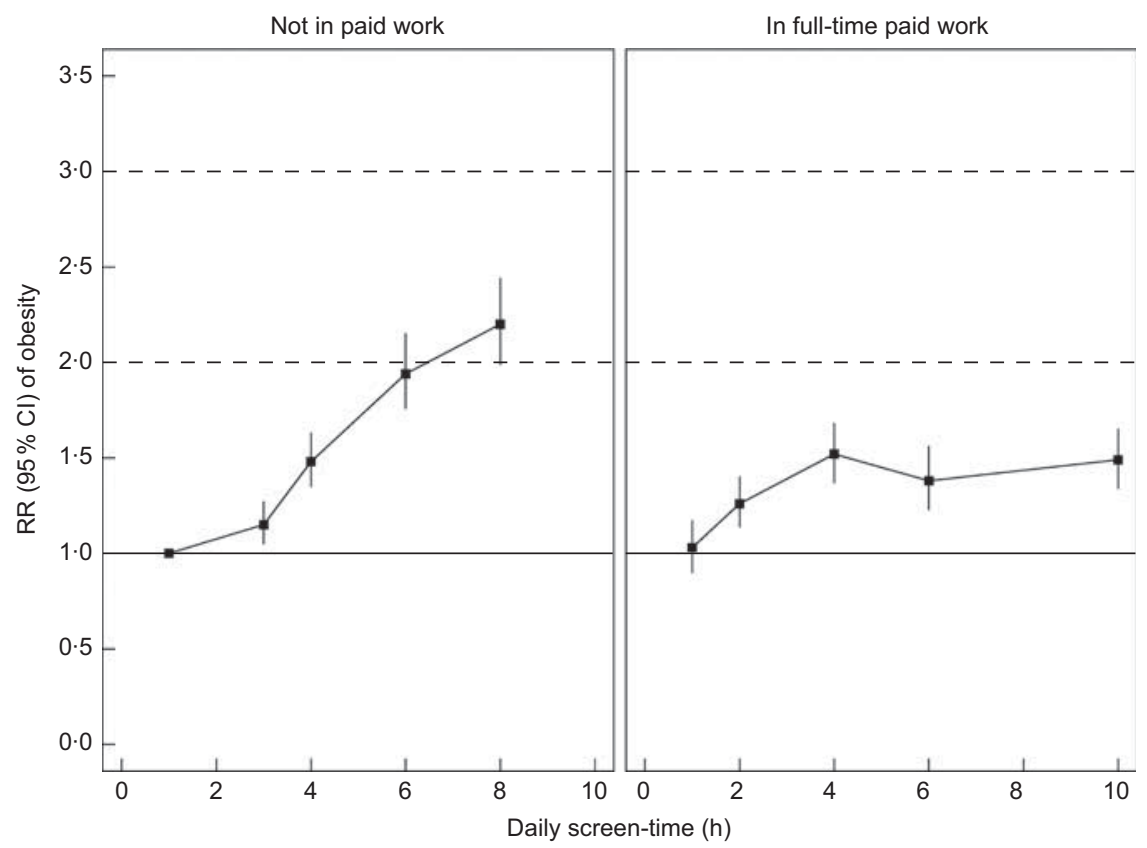

Fig. 2 Relative risk (RR) of obesity according to hours of daily screen-time in study participants who are not in paid work and those in full-time paid work, adjusted for age, sex, income and education (reference group: not in paid work, $<2 \mathrm{~h}$ of daily screen-time); $\mathrm{RR}$ are plotted against the median daily screen-time for each category

\section{Population attributable fraction}

Among people not in paid work, 24\% (95\% CI 21, 27) of obesity could be attributed to daily screen-times of over $2 \mathrm{~h}$. The proportion of obesity in this population attributable to screen-times of $>4 \mathrm{~h} / \mathrm{d}$ was $7 \%(95 \%$ CI 5, 9) among people in full-time work and $13 \%$ (95\% CI 11, 14) among those who were not in paid work. 


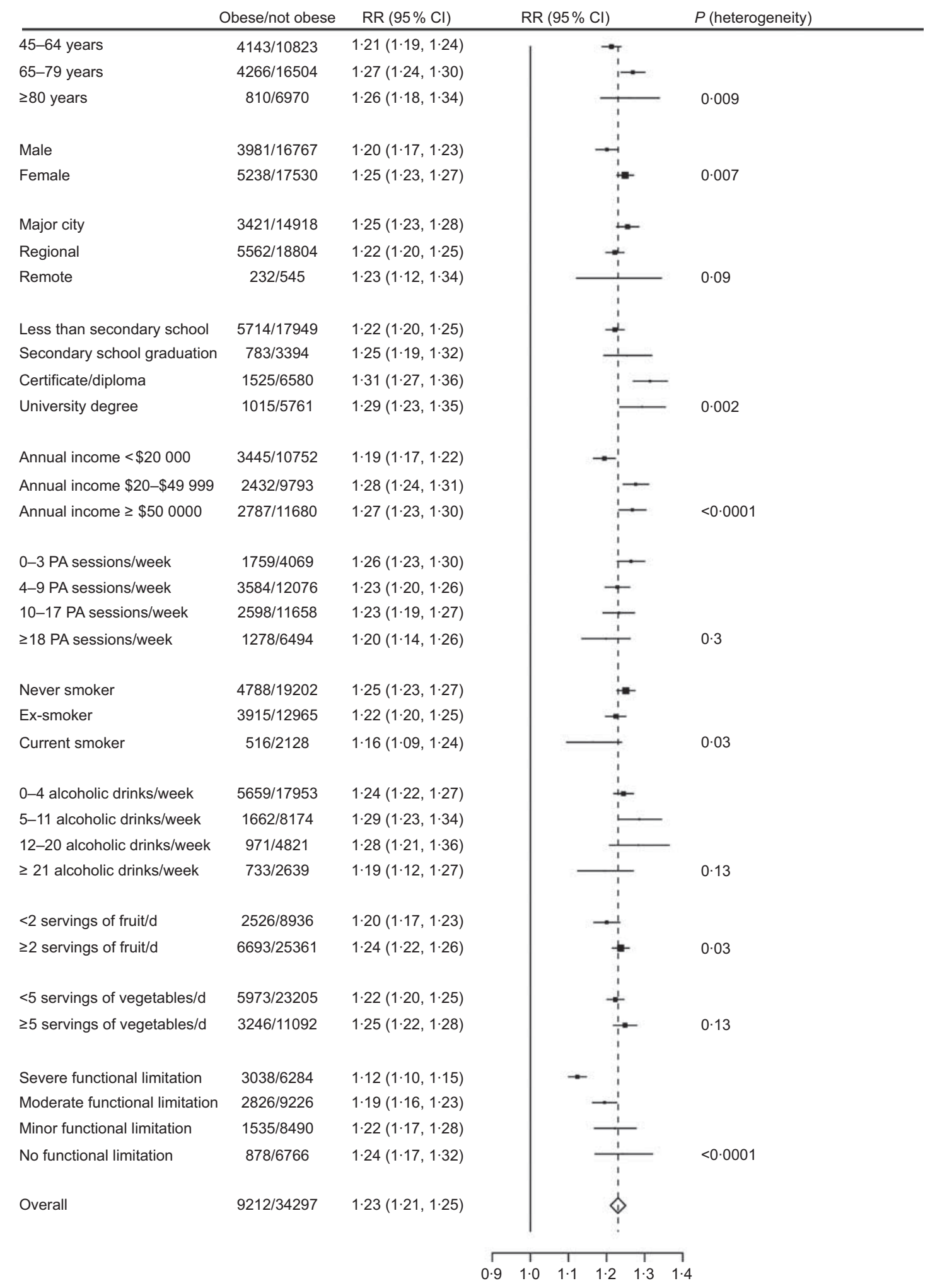

Fig. 3 Relative risk (RR; $95 \% \mathrm{Cl}$ ) of obesity per $2 \mathrm{~h}$ of additional daily screen-time, adjusted for age, sex, income and education, according to various factors

\section{Discussion}

The present study shows that increasing screen-time is associated with increasing risk of obesity, with individuals reporting a screen-time of $>8 \mathrm{~h} / \mathrm{d}$ having a $92 \%$ increase in risk compared to those with $<2 \mathrm{~h} / \mathrm{d}$. Among those not in paid work, there was a $23 \%(95 \%$ CI 21,25$)$ increase in the risk of obesity with every $2 \mathrm{~h}$ of additional daily screen-time, 
and $24 \%(95 \%$ CI 21,27$)$ of obesity in this group could be attributed to screen-times in excess of $2 \mathrm{~h} / \mathrm{d}$.

These findings are consistent with the evidence from previous studies that increasing sedentary activity time, including television watching, is directly related to an increased risk of obesity and overweight ${ }^{(3-7)}$. This relationship is observed in both cross-sectional ${ }^{(3,5,6)}$ and prospective studies $^{(1,4,8)}$. Studies have varied in the way they have measured and categorised screen-time and other sedentary behaviours, as well as obesity-related outcomes, and therefore it is difficult to summarise quantitatively the magnitude of the risk involved. It is interesting to note that our finding of a $23 \%$ increase in the risk of obesity per $2 \mathrm{~h}$ increase in leisure-related screen-time is identical to the $23 \%$ (95\% CI 17, 30) increase per $2 \mathrm{~h}$ of television viewing observed prospectively in the Nurses' Health Study ${ }^{(4)}$.

Individuals with lower levels of overall physical activity were substantially more likely to be obese than those with higher levels of activity. Our findings confirm those of previous studies showing that the effect of screen-time on obesity is independent of the background level of vigorous, moderate or prolonged-walking-related physical activity $^{(3-6)}$. The combined effect of the two variables was substantial, in that $38.3 \%$ of individuals with a screentime of $\geq 8 \mathrm{~h} / \mathrm{d}$ and the lowest physical activity level were obese, and those with the lowest levels of physical activity and highest levels of screen-time had three times the RR of obesity compared to individuals with the highest levels of physical activity and lowest levels of screen-time. Our findings suggest that, in terms of their $\mathrm{RR}$, overall purposeful physical activity and screen-time are of similar importance in relation to obesity.

In keeping with our a priori hypothesis, increasing screen-time among those who were not in paid work had a greater effect on obesity than prolonged screen-time among those in full-time paid work. The reason for this is not known. A possible explanation is that prolonged screen-time in workers is more likely to include prolonged computer use, as well as incidental activity associated with work, whereas prolonged screen-time among those not working might be predominantly television. Alternatively, work- and leisure-related screen-time may be reported with differing degrees of measurement error; overestimation of work-related screen-time would lead to an attenuation of the screen-time-obesity relationship.

Among people not in paid work, significant increases in the RR of obesity with increasing screen-time were observed in all categories of age, physical activity, income, education, region of residence, smoking, alcohol, fruit and vegetable consumption, disability and physical functioning. Screen-time had a significantly attenuated effect among those reporting needing help with day-to-day tasks or with major reductions in functional capacity, compared to other cohort members. It is plausible that those with a major disability have particularly low levels of incidental physical activity and that the metabolic difference between their background levels of incidental activity and distinctly sedentary activities are less marked than for the general population.

Significant but lesser variation in the association between screen-time and obesity was seen according to smoking status, income and education. To our knowledge, the attenuation of the effect of screen-time in smokers has not been shown before, but is consistent with the overall finding of lower BMI in current smokers compared to nonsmokers ${ }^{(20)}$. Why the relationship between screen-time and obesity is attenuated among those with lower income and educational attainment, compared to those with higher income and education, is unclear; it appears to be at least partially explained by greater levels of disability in the lower income and education groups.

Although individuals aged 65 years and above had a lower overall risk of obesity compared to younger individuals, the relationship of increasing obesity with increasing screen-time was similar to that seen in the younger age groups. Men and women experienced similar increases in obesity risk with increasing screentime, as did urban and rural residents.

To our knowledge, the present study describes, for the first time, the relationship between sedentary activities and obesity across the range of middle-aged, old and very old individuals, and also examines the relationship according to the level of disability and a number of other factors. Although the proportions of individuals of healthy weight, overweight and obese in the 45 and Up Study are remarkably similar to those found by objective measurement in the more representative AusDiab Study, the 45 and Up Study is a cohort study and is not designed to be representative of the general population ${ }^{(12)}$. The 'healthy cohort effect' and the relatively low response rates seen in these studies mean that the estimates of RR shown here are likely to be conservative, since community members with more extreme behaviours and health conditions are less likely to participate. However, it is important to note that $\mathrm{RR}$, comparing groups within the cohort, remain valid and can be generalised more broadly ${ }^{(21,22)}$.

Excess body fat is the consequence of sustained positive energy balance, whereby energy intake exceeds energy expenditure. Although dietary factors are implicated in obesity, there is mounting evidence that decreasing physical activity is likely to be a major contributor to the global obesity epidemic ${ }^{(2)}$. Physical activity is often conceptualised as comprised of purposeful and non-purposeful physical activities. The latter is also termed 'incidental' physical activity and this, as well as new components relating to 'non-exercise activity thermogenesis', is increasingly recognised as a contributor to overall energy expenditure ${ }^{(2)}$. Indeed, it has been suggested that differences in incidental physical activity are responsible for the greatest variations in energy expenditure between individuals and populations, accounting for as much as $8368 \mathrm{~kJ}(2000 \mathrm{kcal}) / \mathrm{d}^{(2)}$. 
Our findings highlight the importance of total energy expenditure, rather than solely leisure-time physical activity, for obesity prevention ${ }^{(1)}$, and are consistent with the idea that sedentary behaviours make substantial contributions to reduced overall energy expenditure, mainly resulting from their impact on incidental physical activity, since they may coexist with relatively high levels of purposeful physical activity. Screen-time, particularly television watching, is also associated with other health behaviours, such as eating fatty foods. However, the finding of increased obesity among those watching greater amounts of television persists after adjustment for energy intake ${ }^{(4)}$ and foods eaten while watching television $^{(7)}$, and therefore this is unlikely to explain much of its effects. Prolonged screen-time and sitting time are more common among more highly educated and affluent sections of the population, contrasting with other risk factors for obesity, which tend to be concentrated in lower socio-economic groups.

The data presented here are cross-sectional and therefore do not allow us to exclude the possibility that obesity results in increased sedentary behaviour, rather than the other way around. However, increasing inactivity has been shown to result in increased obesity in longitudinal data ${ }^{(4)}$, and experimental studies show that increasing BMI by overfeeding of lean individuals does not result in increased sedentary behaviour ${ }^{(23)}$. It should be noted that this issue is not resolved completely by using longitudinal data, since the major risk factor for incident obesity is having a high BMI at baseline ${ }^{(23)}$. We suggest that the relationship between sedentary behaviour and obesity is likely to be complex, with a causal relationship between inactivity and obesity likely to predominate. There is likely to be some contribution of obesity leading to inactivity ${ }^{(24)}$, or indeed a 'spiral' relationship, whereby inactivity leads to obesity, which further exacerbates inactivity, leading to further increases in obesity. The present study involved the investigation of a relatively novel variable, combining television and computer use into 'screen-time'. Although this is a useful variable, it does not allow the separation of the effects of television viewing from computer use; further data are being gathered in the 45 and Up Study to allow distinction between these two exposures.

Screen-time, overall physical activity and BMI are all reported with differing degrees of measurement error. A potential explanation of the observation that screen-time is still predictive of obesity within categories of physical activity is that the former measures a similar factor but with less measurement error ${ }^{(5)}$. However, the lack of correlation between screen-time and overall physical activity goes against this argument. It is interesting to note that where physical activity was measured using a pedometer, television viewing remained a significant predictor of obesity in women but not in men, following adjustment for pedometer-measured physical activity ${ }^{(25)}$.

\section{Conclusions}

Current campaigns to reduce overweight and obesity and increase physical activity tend to focus on promoting intermittent purposeful physical activity. The evidence presented here suggests that although this is important, sedentary behaviours such as screen-time and sitting independently and substantially increase the risk of obesity in all of the population groups examined, including the elderly. Hence, public health campaigns to reduce obesity through increasing energy expenditure will be of limited effectiveness unless sedentary behaviours and incidental physical activity are addressed to a similar extent. It is unclear how best to increase incidental physical activity and reduce sedentary behaviours; this is likely to be a fruitful area of future research. Increasing health promotion messages about the need to reduce sedentary television watching, computer use and sitting and to 'move more' may be a first step.

\section{Acknowledgements}

The authors thank the men and women who participated in the 45 and Up Study and Ivan Hanigan for preparing the figures. The 45 and Up Study is funded by The Sax Institute in collaboration with major study partner, the Cancer Council of New South Wales. Other partners are the New South Wales Division of the National Heart Foundation of Australia; the New South Wales Department of Health; beyondblue: the national depression initiative; the New South Wales Department of Ageing, Disability and Home Care; UnitingCare Ageing; and The National Health and Medical Research Council of Australia (Senior Research Fellowship to E.B.). The present study was part of the 45 and Up Study MBF Foundation Policy in Action Roundtable work programme and was also supported by NHMRC Grant 402810; the MBF Foundation is the sole funder of the Policy in Action Roundtable. The authors declare no conflict of interest. E.B., L.J. and A.B. were responsible for the conception and design of the study; E.B. and L.J. collected the data; K.R. and M.C. performed the data analysis and given statistical advice; E.B. and M.C. supervised the analysis; and E.B., L.J., K.R., M.C. and A.B.were responsible for drafting and approval of the final manuscript.

\section{References}

1. Bauman A, Allman-Farinelli M, Huxley R et al. (2008) Leisure-time physical activity alone may not be a sufficient public health approach to prevent obesity - a focus on China. Obes Rev 9, 119-126.

2. Levine JA (2007) Nonexercise activity thermogenesis liberating the life-force. J Intern Med 262, 273-287.

3. Salmon J, Bauman A, Crawford D et al. (2000) The association between television viewing and overweight among Australian adults participating in varying levels of leisure-time physical activity. Int J Obes 24, 600-606. 
4. Hu FB, Li TY, Colditz GA et al. (2003) Television watching and other sedentary behaviors in relation to risk of obesity and type 2 diabetes mellitus in women. JAMA 289, 1785-1791.

5. Jakes RW, Day NE, Khaw KT et al. (2003) Television viewing and low participation in vigorous recreation are independently associated with obesity and markers of cardiovascular disease risk: EPIC-Norfolk population-based study. Eur J Clin Nutr 57, 1089-1096.

6. Sugiyama T, Healy GN, Dunstan DW et al. (2008) Joint associations of multiple leisure-time sedentary behaviours and physical activity with obesity in Australian adults. Int J Behav Nutr Phys Act 5, 35.

7. Cleland VJ, Schmidt MD, Dwyer T et al. (2008) Television viewing and abdominal obesity in young adults: is the association mediated by food and beverage consumption during viewing time or reduced leisure-time physical activity? Am J Clin Nutr 87, 1148-1155.

8. Ball K, Brown W \& Crawford D (2002) Who does not gain weight? Prevalence and predictors of weight maintenance in young women. Int J Obes 26, 1570-1578.

9. Martinez-González MA, Martinez JA, Hu FB et al. (1999) Physical inactivity, sedentary lifestyle and obesity in the European Union. Int J Obes Relat Metab Disord 23, 1192-1201.

10. Stamatakis E, Hirani V \& Rennie K (2008) Moderate-tovigorous physical activity and sedentary behaviours in relation to body mass index-defined and waist circumference-defined obesity. Br J Nutr 101, 765-773.

11. Tudor-Locke C, Burton NW \& Brown WJ (2009) Leisuretime physical activity and occupational sitting: associations with steps/day and BMI in 54-59 year old Australian women. Prev Med 48, 64-68.

12. 45 and Up Study Collaborators (2008) Cohort profile: the 45 and Up Study. Int J Epidemiol 37, 941-947.

13. Australian Institute of Health and Welfare (2004) Rural, Regional and Remote Health: A Guide to Remoteness Classifications. AIHW Catalogue PHE no. 53. Canberra: AIHW; available at www.aihw.gov.au
14. Australian Institute of Health and Welfare (2003) The Active Australia Survey: A Guide and Manual for Implementation, Analysis and Reporting. Canberra: AIHW.

15. Bauman A, Ford I \& Armstrong T (2001) Trends in Population Levels of Reported Physical Activity in Australia 1997, 1999 and 2000. Canberra: Australian Sports Commission.

16. Australian Institute of Health and Welfare (2003) The Active Australia Survey: A Guide for Implementing Analysis and Reporting. Catalogue no. CVD 22. Canberra: AIHW.

17. World Health Organization (2000) Obesity: Preventing and Managing the Global Epidemic. Report of a WHO Consultation. WHO Technical Report Series no. 894. Geneva: WHO.

18. Blizzard L \& Hosmer D (2006) Parameter estimation and goodness-of-fit in log binomial regression. Biom J48, 5-22.

19. Rothman N, Greenland S \& Lash T (2008) Modern Epidemiology. Philadelphia, PA: Lippincott, Williams and Wilkins.

20. Brown A \& Siahpush M (2007) Risk factors for overweight and obesity: results from the 2001 National Health Survey. Public Health 121, 603-613.

21. Ponsonby A-L, Dwyer T \& Couper D (1996) Is this finding relevant? Generalisability and epidemiology. Aust $N Z J$ Public Health 20, 54-56.

22. Mealing N, Banks E, Jorm L et al. (2010) Investigation of relative risk estimates from studies of the same population with contrasting response rates and designs. BMC Medical Research Methodology 10, 26.

23. Levine JA, Lanningham-Foster LM, McCrady SK et al. (2005) Interindividual variation in posture allocation: possible rote in human obesity. Science 307, 584-586.

24. Ekelund U, Brage S, Besson $\mathrm{H}$ et al. (2008) Time spent being sedentary and weight gain in healthy adults: reverse or bidirectional causality? Am J Clin Nutr 88, 612-617.

25. Dwyer T, Hosmer D, Hosmer T et al. (2007) The inverse relationship between number of steps per day and obesity in a population-based sample - the AusDiab study. Int $J$ Obes 31, 797-804. 